\title{
Simple Synthesis of Fluorophore Using Water-Soluble Chitosan Oligomer
}

\author{
Hun Min Lee, Ja Young Cheon, Seoho Lee, Seung Hyun Lee, Su Young Kim, Dong Hoon Kang, Jeong Woo Heo and \\ Won Ho Park ${ }^{*}$ \\ Department of Advanced organic materials and Textile System Engineering, Chungnam National University, Daejeon, Korea \\ *Corresponding author
}

\begin{abstract}
In the past few decades, a great interest has been focused on a naturally occurring class of polymers called chitosan for their large amount in nature, biodegradability and extensive applications. Water-soluble chitosan oligomer is composed of $\beta$ (1,4)-2-amido-2-deoxy-D-glucan and $\beta$-(1,4)-2-acetoamido-2deoxy-D-glucan(acetylglucosamine), and the low molecular weight substances obtained by acidic or enzymatic hydrolysis of chitosan. Until now, many researchers have examined chitosan oligomer as a promising material for biomedical applications on account of its good biocompatibility, biodegradability, antimicrobial activity and wound healing effects. Dye-labeled chitosan can be also employed in bio-imaging system, because it has low toxicity. Some dyes, such as Alexa Fluor, Cibacron Blue and fluorescein isothiocyanate (FITC), have been employed to create dye-labeled chitosan particulate systems. However, a greater part of approaches have been developed for the synthesis of dye-labeled chitosan molecular system using chemical reagents due to its low solubility. Numerous researches have addressed the utilization of chitosan as a fluorescence probe. These methods have a limited application in the medical and pharmaceutical fields, because most of them may be environmentally toxic or biologically hazardous. Herein, we focused on the environmentally friendly approach using a water-soluble chitosan derivative for bio-imaging. We report on the simple synthesis and optical properties for FITC-labeled chitosan oligomer in distilled water.
\end{abstract}

Keywords-chitosan oligomer; silver chloride nanoparticle; fluorescent

\section{INTRODUCTION}

Nowadays, fluorescent natural polymers are interested because of their promising applications in biological/biomedical labeling, tracking, monitoring, imaging, and diagnostics, particularly in drug delivery systems, tissue engineering and cancer imaging. Although a unique feature of natural polymers compared with synthetic ones is their ability to undergo biodegradation, most of the studies thus far have been carried out with synthetic polymers.

Organic fluorophores can be attached to the polymer chains through covalent or non-covalent linkages and exhibit fluorescence from short to very long wavelengths, depending on their nature. The synthesis of fluorescent polymers is generally carried out using two established methods. The first is through the polymerization of fluorescent monomers, and the second is via the attachment of fluorescent moieties to the polymer backbone. To preserve the properties of the natural polymer, the latter method is often more useful. Derivatization of polymer backbones, in this case, addition of fluorophores, is often done to enable them to be used to meet a specific function, and this is often monitored by a specific detection event, such as fluorescence or absorption in the visible region, or at a long wavelength. Reagents used for derivatization may be divided into four groups: non-fluorescent reagents, fluorogenic reagents that are generally non-fluorescent but that react with target compounds to form conjugated fluorescent cyclic molecules, fluorescent reagents having a highly fluorescent aromatic group (fluorophore), and redox active reagents that are often employed in electrochemistry.

Chitosan (CHI) is a natural polymer consisting of 1.4linked N-acetyl-D-glucosamine (GlcNAc) and D-glucosamine (GlcN) subunits. CHI is nontoxic, biocompatible, and biodegradable and has mucoadhesive properties; therefore, it has attracted significant interest in a broad range of scientific areas, particularly in biomedical and pharmaceutical research. Recently, various fluorescent CHI derivatives were synthesized by covalently attaching fluorescent groups such as fluorescein isothiocyanate (FITC), rhodamine B isothiocyanate, naphthalimide derivatives, 1-pyrenebutyric acid N-hydroxyl succinamide ester (PSE), xanthene dye Rose Bengal, Rose Bengal, 1-naphthylacetic acid, anthracene-9carboxaldehyde, anthracene-9-carboxylic acid, 2-(2'hydroxyphenyl)- benzoxazole, 18 dansyl chloride, and $\mathrm{N}$ hydroxy-succinimide functionalized cyanine and cyanine to the primary amino groups in CHI. Moreover, fluorescent CHI nanoparticles were prepared for use as nanoprobes or nanocoating for sensing, imaging, or bone repair in biological applications.

Herein, we focused on the environmentally friendly approach using a water-soluble chitosan derivative for bioimaging. We report on the simple wynthesis and optical properties for FITC-labeled chitosan oligomer (CHI-FITC) in distilled water.

\section{EXPERIMENTAL}

\section{A. Simple Synthesis of CHI-FITC Using Distilled Water}

CHI-FITC having various degrees of FITC chromophore substitution were prepared. The derivatives were synthesized through reaction of the amine groups in CHI with the FITC isothiocyanate groups in ethanol. The general procedure was as follows: $\mathrm{CHI}(0.6 \mathrm{~g})$ was dissolved in $2 \%(\mathrm{w} / \mathrm{v})$ water $(30$ $\mathrm{mL}$ ) at room temperature with stirring. After complete $\mathrm{CHI}$ dissolution, a solution of FITC $(10,30$ and $50 \mathrm{mg})$ in $100 \mathrm{~mL}$ 
of ethanol was added to the CHI solution. The mixture was intensively stirred using a magnetic stirring bar for $24 \mathrm{~h}$ with protection from light. After the reaction was completed, the mixture was centrifugated (4,000 rpm, $10 \mathrm{~min}$ ) and washed by ethanol. The degree of N-substitution (DS) of FITC units in the derivatives was determined using $1 \mathrm{H}$ NMR spectroscopy. CHI-FITC with different DS values $(0.01,0.03,0.04)$ are subsequently represented as CHI-10FITC, CHI-30FITC and CHI-50FITC respectively.

\section{RESUlTS AND DISCUSSION}

The composition of FITC-CHI was determined by FT-IR and $1 \mathrm{H}$ NMR. CHI-FITC exhibits the characteristic bands of benzene ring vibrations at 1458,1535 , and $1593 \mathrm{~cm}-1$, while the $\mathrm{N}=\mathrm{C}=\mathrm{S}$ (isothiocyanate) vibrations of FITC disappeared at $2038 \mathrm{~cm}-1$ due to the aminosilanization reaction. The characteristic $\delta$ value of aromatic proton form from 6.3 to 8 ppm appeared, and the degree of substitution (DS) was increased with the increase of the FITC concentration. The maximum fluorescence emission exhibited at $528 \mathrm{~nm}$ when the excitation wavelength was set at $400 \mathrm{~nm}$, and also increased with the increase of the DS.

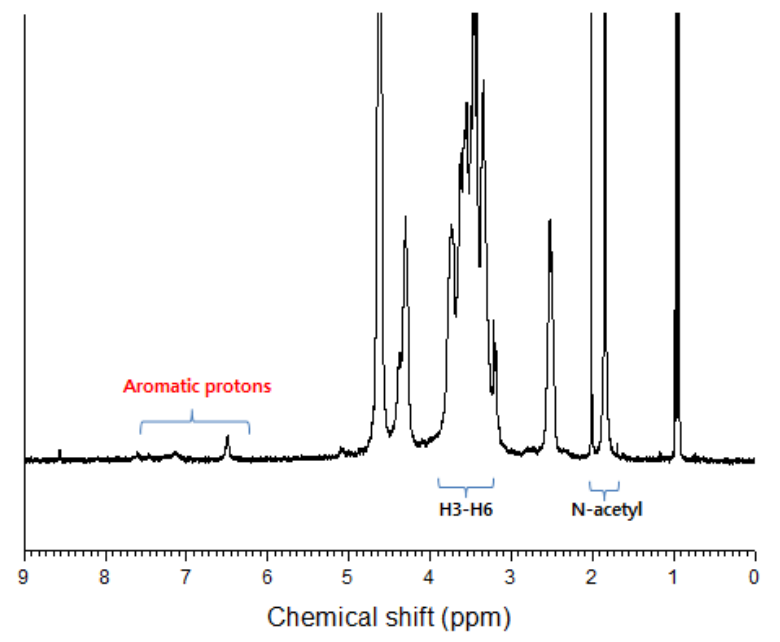

FIGURE I. ${ }^{1} \mathrm{H}$ NMR SPECTRA OF CHI-50FITC

\section{CONCLUSION}

In this work, we synthesized FITC-labeled chitosan oligomer as $\mathrm{s}$ water-soluble fluorophore by environmentally friendly method using distilled water. The composition of FITC-CHI was determined by FT-IR and $1 \mathrm{H} \mathrm{NMR}$ and the degree of substitution (DS) was increased with the increase of the FITC concentration. CHI-FITC is reacted with silver nitrate to form the silver nanoparticles and the intensity of fluorescence was quenched because FITC was changed to fluorescence resonance energy transfer (FRET) by forming silver nanoparticles. In the efforts to use the distilled water as a reaction solvent, the simple process to prepare FITC-labeled $\mathrm{CHI}$ oligomer using benign condition is expected to be providing numerous benefits and compatibility for pharmaceutical, bio-medical, bio-sensing applications. And we will investigate the change of fluorescence intensity at various experimental conditions such as $\mathrm{pH}$, temperature, and ratio of reactants.

\section{REFERENCES}

[1] G. Eason, B. Noble, and I. N. Sneddon, "On certain integrals of Lipschitz-Hankel type involving products of Bessel functions," Phil. Trans. Roy. Soc. London, vol. A247, pp. 529-551, April 1955. (references)

[2] O. P. Siwach, and P. Sen, "Fluorescence properties of Ag nanoparticles in water," Solid State Communications, Spectrochimica Acta Part A, vol. 129, pp. 659-663, January 2009.

[3] P. Gonil, and S. Puttipipatkhachorn, "Synthesis and Fluorescence Properties of N-Substituted 1-Cyanobenz[f]isoindole Chitosan Polymers and Nanoparticles for Live Cell Imaging," Biomacromolecules, vol. 15, pp. 2879-2888, June 2014.

[4] C. A. sabatini, and M H. Gehlen, "Fluorescence Modulation of Acridine and Coumarin Dyes by Silver Nanoparticles," Journal of Fluoresence, vol. 17, pp. 377-382, July 2007.

[5] Z. Chen, C. Zhang, Y. Tna, T. Zhou, H. Ma, C. Wan, Y. Lin, and K. Li, "Chitosan-functionalized gold nanoparticles for colorimetric detection of mercury ions based on chelation-induced aggregation," Microchimica Acta, vol. 182, pp. 611-616, September 2014. 